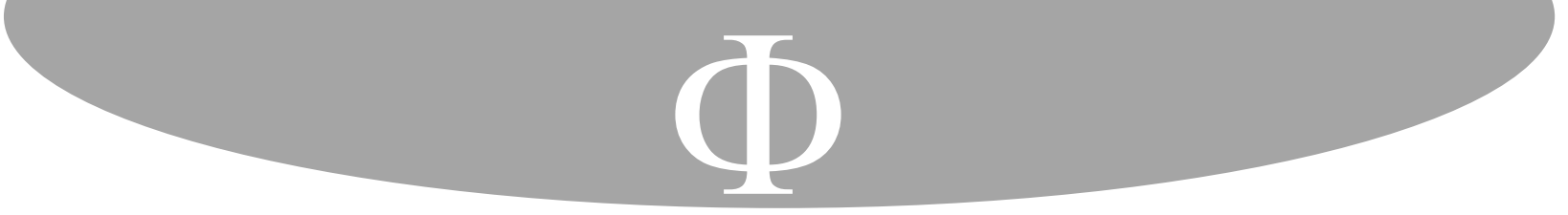

\title{
Plotino y la novedad de su principio metafísico*
}

\begin{abstract}
Fernando Gabriel Martin De Blassi**
Universidad Nacional de Cuyo

Mendoza, Argentina

Para citar este artículo: Martin De Blassi, Fernando Gabriel. «Plotino y la novedad de su principio metafísico». Franciscanum 176, Vol. 63 (2021): 1-25.

\section{Resumen}

Las historias de la filosofía consideran que la doctrina de Plotino inaugura el tercer período de la escuela platónica, denominado con la categoría historiográfica de neoplatonismo. Sobre la base de este presupuesto, que organiza la tradición del pensamiento de acuerdo con una sucesión cronológica de las ideas y de sus respectivas influencias, el presente trabajo intenta hacer valer una distinción crítica a propósito de la originalidad metafísica del principio plotiniano. En virtud de un análisis favorecido por los textos fuentes y por la bibliografía erudita, se pretende mostrar que la trascendencia absoluta del Uno-Bien respecto del mundo corresponde en rigor a un orden de verdades mucho más cercano al derrotero filosófico de la fase subsiguiente.
\end{abstract}

\section{Palabras clave}

Historia de la filosofía, Metafísica, Plotino, Uno-Bien, Neoplatonismo.

\section{Plotinus and the novelty of his metaphysical principle}

\footnotetext{
* El presente trabajo corresponde a una instancia de investigación de un proyecto más amplio (Cód. 38/2016, periodo 2019-2022), acreditado por la Agencia Nacional para la Promoción Científica y Tecnología, cuyo titulo reza: «Los presupuestos teóricos del mindfulness (atención plena a lo real) o MBTC en la terapia de la depresión y otros desordenes de la afectividad». El proyecto mentado está dirigido por el Dr. Rubén Peretó Rivas y se ejecuta dentro de un equipo de investigación interdisciplinario, financiado por el Consejo Nacional de Investigaciones Científicas y Técnicas de Argentina (CONICET).

** Prof., Lic. y Dr. en Filosofía en Filosofía por la Facultad de Filosofía y Letras de la Universidad Nacional de Cuyo. Su tesis doctoral fue escrita bajo la dirección del Prof. Dr. Martín Zubiria y versó sobre «La procesión hipostática y el ingreso en el sí-mismo en la doctrina de Plotino sobre la diferencia absoluta: contribuciones teóricas para comprender la originalidad de su posición metafísica». Se desempeña actualmente como docente del Departamento de Filosofía de la Unidad Académica mencionada y como becario posdoctoral del Consejo Nacional de Investigaciones Científicas y Técnicas de Argentina. El grupo de investigación al que pertenece se denomina: «Centro de Estudios Filosóficos Medievales» (CEFIM), que forma parte a su vez del Instituto de Filosofía de la Facultad de Filosofía y Letras-UNCuyo. ORCID: https://orcid.org/0000-0003-0767-4705. Contacto: martindeblassi@ffyl.uncu.edu.ar; martindeblassi@gmail.com.
} 


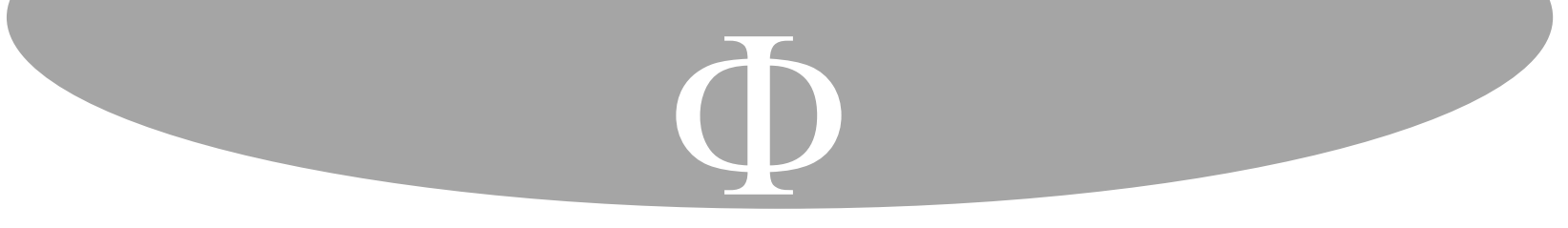

En el marco de este dilema entre mística o exégesis ${ }^{61}$, una línea interpretativa considera que la experiencia del propio Plotino en relación con el ascenso extático determina en gran medida su quehacer filosófico ${ }^{62}$. Con respecto a este punto, se aduce que el rigor dialéctico seguido por Plotino para dilucidar algún problema exegético no sería ya lo fundamental de su propio genio reflexivo, sino que ello sería, antes bien, el fruto de un momento previo de profunda intimidad unitiva ${ }^{63}$. Durante ese instante, por lo general breve y repentino, el yo personal no está sujeto a las solicitudes exteriores, sino que se encuentra disponible para captar una «belleza maravillosa» $(\theta \alpha v \mu \alpha \sigma \tau o ̀ v ~(. . .) \kappa \alpha ́ \lambda \lambda o \varsigma)^{64}$, cuya luz no

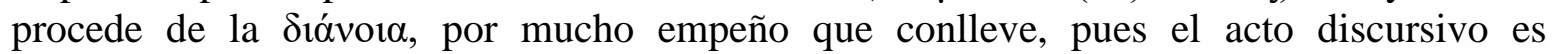

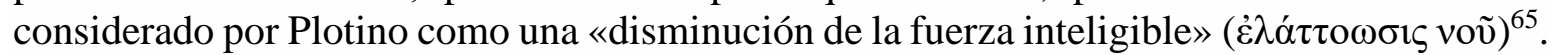
Para los especialistas que adhieren a esta postura, el contacto con la presencia efectiva del Uno-Bien deja una marca tan indeleble en la vida del hombre que la erudición del entendimiento queda relegada a un segundo plano. En el extremo opuesto, están quienes defienden la hermenéutica contraria, alegando que se puede prescindir de la experiencia mística puesto que la exégesis propiciada por Plotino no está vinculada lógica y necesariamente con aquella ${ }^{66}$. Sea de ello lo que fuere, no hay motivos suficientes para que haya un antagonismo entre las dos posiciones. Ambas pueden coexistir equilibradamente sin por ello atentar contra la unidad del pensamiento plotiniano. De hecho, los textos evidencian que es uno y el mismo autor quien reflexiona metódicamente a la vez que exulta de entusiasmo en ciertos pasajes de su obra, cuando está por finalizar la investigación acometida $^{67}$.

En otro orden de consideraciones, se advierte que tanto la exégesis como la mística son canales de retroalimentación a lo largo del recorrido anagógico trazado por Plotino: a mayor intimidad contemplativa, mayor sed de sabiduría y a mayor esfuerzo exegético, mayor claridad en la fruición espiritual ${ }^{68}$. Así las cosas, la propuesta filosófica de Plotino es la propia de un «místico practicante» porque no se reduce a una enseñanza de cuño teórico, en la medida en que discurre sobre la posibilidad de una unión con el principio además de haberla experimentado $^{69}$. Ello es que la doctrina de Plotino no sólo busca reflexionar acerca del ascenso cognoscitivo, sino que requiere también de una «decisión» para «aunarse con lo

\footnotetext{
${ }^{61}$ Cf. G. Martino, «Mística y exégesis en la filosofía de Plotino», NOVA TELLVS 2, Vol. 30 (2012): 73-98.

${ }^{62}$ Porfirio confesó que durante su estancia en la escuela de Plotino, el maestro había alcanzado cuatro veces una elevación de tenor místico merced a una actividad inefable (Porfirio y Plotino, Vida de Plotino y Enéadas, 23, 15-17). También, en dos oportunidades, el filósofo apela a su experiencia personal para dar crédito al

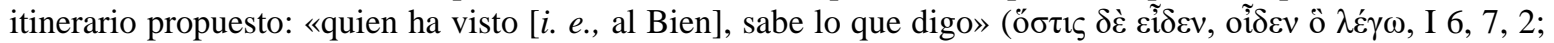
VI 9, 9, 46-47).

${ }^{63}$ Cf. Th. A. Szlezák, Platon und Aristoteles in der Nuslehre Plotins (Basel-Stuttgart: Schwabe \& Co, 1979), $9-51$.

${ }^{64}$ IV $8,1,3$.

${ }^{65}$ IV $3,18,4$.

${ }^{66}$ Cf. Ll. P. Gerson, Plotinus (London: Routledge, 1994), 220 ss.

${ }^{67}$ Cf. VI 7, 34, 9-37.

${ }^{68}$ Cf. P.-Sh. Ho, Plotinus' Mystical Teaching of Henosis. An Interpretation in the Light of the Metaphysics of the One (Frankfurt a. M.: Peter Lang, 2015).

${ }^{69} \mathrm{Cf}$. Dodds, Paganos y cristianos en una época de angustia, 100.
} 


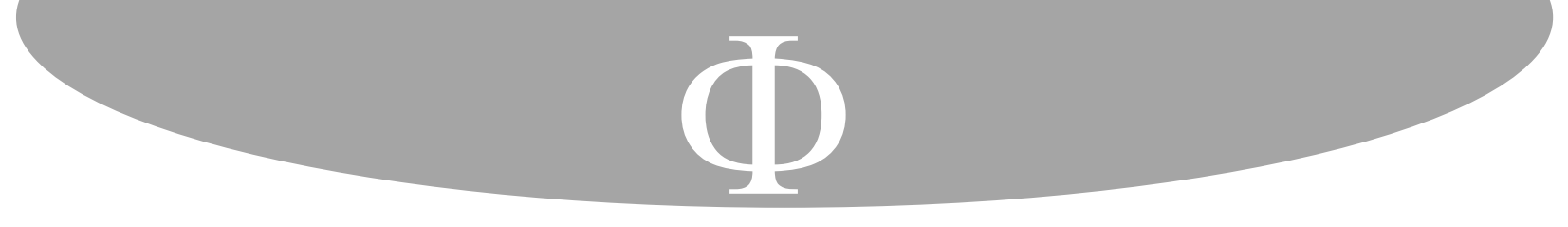

Sin duda alguna, cabe señalar que ya antes de Plotino se aprecia una tendencia dirigida a fijar en la unidad un principio supremo. Esta tendencia se halla influida sobre todo por la concepción platónica de la idea del Bien - como aquella idea que está «más allá de la esencia»

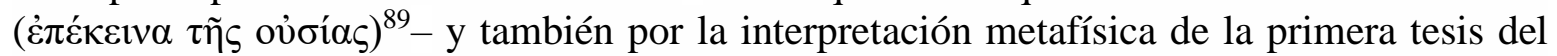
Parménides de Platón ${ }^{90}$. Pese a esto, la comprensión del principio primero como Uno transcendente a la sustancia inteligible no logra en los antecesores a Plotino la claridad argumentativa de que sí goza en este autor ${ }^{91}$. En efecto, ciertos discursos sobre el estatuto del principio metafísico último, pertenecientes al platonismo medio ${ }^{92}$ o bien a la gnosis ${ }^{93}$, hablan del Uno o del Bien por medio de las cualidades que atañen sólo a la inteligencia o bien a la esencia. En cambio, el Uno-Bien plotiniano se encuentra por encima de aquellas realidades ${ }^{94}$.

De acuerdo con lo dicho, Plotino considera que el Uno-Bien es un principio de suyo ajeno al nivel subsistente, aun cuando empero genere y sostenga las hipóstasis que vienen

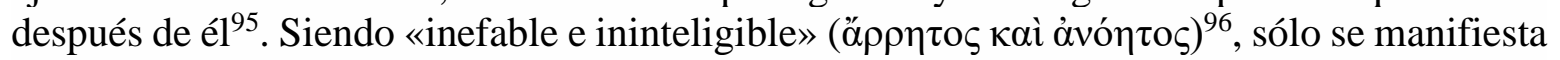
a través del «vestigio» que imprime en lo diverso ('í $\chi$ vo $\varsigma<\tau o \tilde{v}>\dot{\varepsilon} v o ́ \varsigma){ }^{97}$. De esta manera, cada ser ostenta una traza del Uno sin agotarlo ${ }^{98}$. La unidad se puede intensificar o aminorar, en la medida en que se aproxime a su principio o se aleje de él ${ }^{99}$. En el caso del alma racional, la semejanza primera se hubo perdido una vez que el alma descendió ${ }^{100}$. Pero ella tiene la

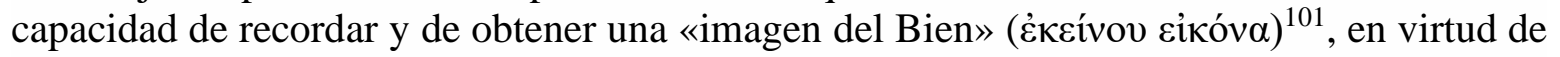
la cual puede ascender y recobrar su estado de pureza original. Esta conversión no se presenta bajo la forma de un volverse hacia lo otro de sí misma sino que cabe designarla, con mayor exactitud, como un ingreso desde su ser-otro hacia el sí-mismo que ya era cuando estaba cabe el Bien. La garantía última del acceso a la presencia del Uno-Bien no depende en rigor del individuo singular, sino del propio «don» $(\tau \text { ò } \delta i \delta \text { òv })^{102}$, que este recibe para tomar parte en la «semejanza» $(\dot{o} \mu o i ́ \omega \sigma ı))$ con aquel ${ }^{103}$.

\footnotetext{
${ }^{89}$ Resp. 509 b.

${ }^{90}$ Cf. E. R. Dodds, «The Parmenides of Plato and the Origin of the Neoplatonic One», The Class. Quarterly, 34, Vol. 22 (1928): 129-142.

${ }^{91}$ Cf. M. L. Gatti, «Plotinus: The Platonic Tradition and the foundation of Neoplatonism», en The Cambridge Companion to Plotinus, ed. Ll. P. Gerson (Cambridge: University Press, 1999), 10-37.

92 Cf. H. Dörrie, «Die Frage nach dem Transzendenten im Mittelplatonismus», en Les sources de Plotin. Entretiens sur l'Antiquité Classique V, ed. E. R. Dodds (Genève: Fondation Hardt, 1960), 193-241.

${ }^{93}$ Cf. A.-J. Festugière, La Révélation d' Hermès Trimégiste. IV: Le Dieu Inconnu et la Gnose (Paris: Les Belles Lettres, 1986), 92-140.

${ }^{94}$ Cf. J. Whittaker, «EПEKEINA NOY KAI OY IIAS», Vigilia Christianae 2, Vol. 23 (1969): 91-104.

${ }^{95}$ Cf. Szlezák, Platon und Aristoteles in der Nuslehre Plotins, 193-241; J. Bussanich, The One and its relation to Intellect in Plotinus (Leiden-New York: Brill, 1988), 2-3, 27-32.

${ }^{96} \mathrm{~V} \mathrm{3}, 10,43$.

${ }^{97}$ V 5, 5, 14; cf. J. H. Sleeman \& G. Pollet, Lexicon Plotinianum (Leiden-Leuven: Brill, 1980), s. v. ǐ $\chi v 0 \varsigma, 517$, 43 ss.

${ }^{98}$ Cf. V 5, 5, 1-14.

${ }^{99}$ Cf. V 5, 4, 1-10.

${ }^{100}$ Cf. D. O’ Brien, «Le volontaire et la nécessité : réflexions sur la descente de l'âme dans la philosophie de Plotin», Revue Philosophique de la France et de l'Étranger, 4, Vol. 167 (1977): 401-422.

101 VI 9, 11, 7.

102 VI 5, 10, 31.

${ }^{103}$ Cf. I 2, 5, 2; 6, 26.
} 


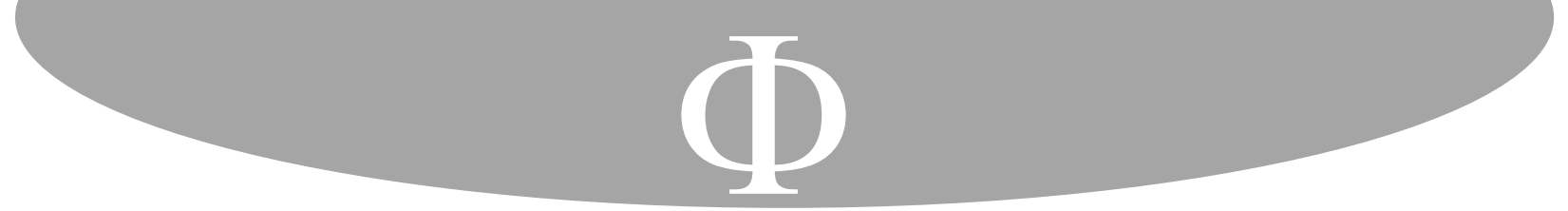

De todos los seres, el alma humana goza de una particular condición, puesto que ella puede acrecentar o aminorar el grado de participación con respecto a la fuente de unidad. Sólo que su decisión se enmarca dentro de una libertad mayor, referida a la voluntad del mismo Uno-Bien. Este principio no está sujeto ni siquiera a la generación de los seres. La lejanía y ausencia de cada individuo no hacen mella a la trascendencia del Primero; antes bien, son los seres quienes tienen en sus manos la posibilidad de alejarse de la bondad o de aproximarse a ella en virtud de la propia decisión. En la medida en que el alma singular alberga en sí misma el recuerdo inteligible, puede remontarse hacia la belleza de lo alto. Estando allí, en la fruición del verdadero objeto de amor, el alma se vuelve semejante a quien le concedió la vida y el ser. De modo tal que la filosofía de Plotino no finaliza en lo irracional, sino que se apoya en lo inteligible para sobrepasarlo. Al revocar el propio disfrute que implica al hombre conocerse a sí-mismo, se puede acceder a la dicha de una vida completa ${ }^{104}$. De esta manera, el encuentro con el Uno-Bien nunca se logra por un deseo curioso del alma singular, sino sólo en la medida en que el Primero le permite entrar en contacto con su

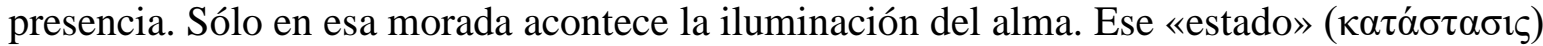
es definido por el filósofo como «resultado de la actividad espiritual» (̇̉vé $\rho \gamma \varepsilon \mu \alpha$ ), un estado

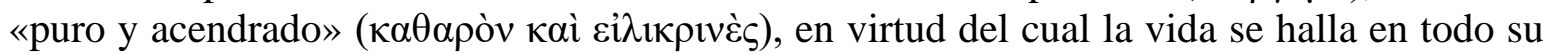

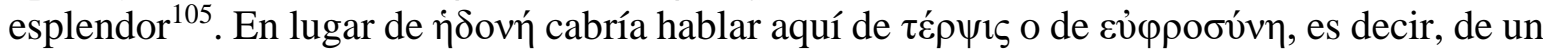
deleite suscitado a raíz del gozo espiritual, que el griego neotestamentario caracteriza con el nombre de $\chi \alpha \rho \alpha ́$. Se trata de una experiencia análoga a la que narra San Agustín en Confesiones, libro VII, tras haberle sido revelada una luz pura que estaba por encima de su mente y en virtud de cuya unión era dable a su alma estar en la verdad y en un gozo indecible, por haberse liberado de toda utilidad con respecto al fin ${ }^{106}$.

En consecuencia, Plotino considera que el hombre puede ser iluminado por los inteligibles aun cuando se haya encarnado. Ello explica que, para Plotino, el destino del alma no sea lo más íntimo sino, en rigor, lo más elevado ${ }^{107}$. La interioridad se determina como un término medio que permite vincular lo de aquí con lo de allí, en la unidad de una contemplación que se va volviendo tanto más unificada cuanto más íntima y penetrante es la claridad del objeto contemplado. Una vez que el alma se alejó por el deseo de mandar, perdió las alas y descendió. Sin embargo, ella porta en su interior la huella del Primero, jamás descenderá hasta el no ser total. Quizá descienda hasta el mal, esto es, la materia, pero nunca

\footnotetext{
${ }^{104}$ Cf. J. Croissant, «P. Henry S.J. Le problème de la liberté chez Plotin, dans Revue néoscol. de philos., XXXIII, févr. mai et août 1931, pp. 50-79, 180-215, 318-339», L'Antiquité Classique Vol. 4, 2 (1935): 535: «Il n’y a liberté que dans la conversion. Cette conception profonde, originale est exprimée dans le traité sur La liberté et la volonté de l'Un, dont l'auteur donne une bonne analyse. Il s'en dégage une notion de la liberté absolument nouvelle, rompant avec la tradition hellénique qui ne conçoit la liberté qu'au regard de l'action. Pour Plotin, la liberté n'est pas au principe de l'action vertueuse, elle est au terme de l'ascèse intérieure par laquelle, grâce à la vertu, l'âme se dégage du monde sensible pour vivre selon l'Intelligence. La liberté est introversion, aspiration au Bien, amour de Dieu».

${ }^{105} \mathrm{Cf}$. VI 7, 30, 22

${ }^{106}$ Cf. S. Agustín, Confesiones, est. prel., trad. y notas de S. Magnavacca (Buenos Aires: Losada, 2011), X, 16, p. 191.

${ }^{107}$ Cf. II 9, 16, 48-49; VI 5, 7, 1 ss; 10, 7-9; VI 7, 17, 27.
} 


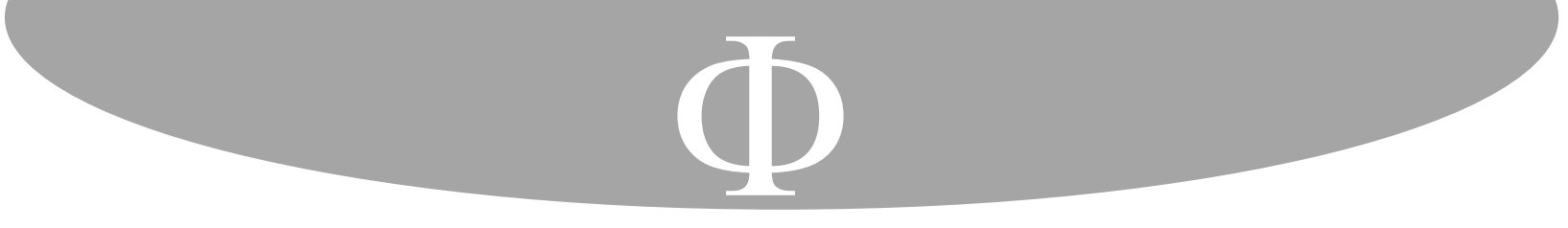

Uno primero, bien que no bajo la forma de algo sino de alguien, pues el Uno es el Bien que se revela como el origen de toda otra procedencia ${ }^{156}$.

El tránsito de una verdad reconocida intelectualmente a una contemplación que cobra la forma de una operatio - puesto que mueve al amor de unión- evidencia otro ámbito histórico y filosófico que ya no responde al modo griego de comprender la naturaleza metafísica de la verdad. Ahora bien, ¿de qué operatio se trata? Según se mire, desde el punto de vista del principio ella atañe al poder del Uno-Bien que, no obstante su naturaleza trascendente, ha permitido la subsistencia de lo múltiple. Desde el punto de vista del alma humana, en cambio, la operatio concierne a la negación del propio límite para ser definitivamente sí-mismo, esto es, la unión en la intimidad de visión y espectáculo. De allí que los términos plotinianos de contemplación y de operatio se conjuguen mutuamente en una misma trama semántica, que apunta al logro de una plenitud en el «despojamiento» del hombre envilecido ( $\alpha \dot{\varphi} \varphi \lambda \varepsilon \varepsilon \alpha \dot{\alpha} v \tau \alpha)^{157}$.

\section{A modo de conclusión}

Para finalizar, cabe señalar que el principio trascendente de la metafísica de Plotino representa un novum en la historia de la filosofía por el modo de fundamentar la esencia de la verdad. La intelección plotiniana se concibe como la primera respuesta racionalmente satisfactoria a la irrupción histórica sin precedentes de la trascendencia divina ${ }^{158}$, de cuya verdad esencialmente operativa dependerán en buena medida las posiciones posteriores ${ }^{159}$. De lo dicho cabe inferir que, aun cuando Plotino se haya ocupado una y otra vez en la fílosofía precedente, no buscó empero asilo en ella. Por lo tanto, se debe afirmar que el pensamiento plotiniano produce una innovación radical con respecto al platonismo, pese a que Plotino recurra con insistencia a los textos del fundador de la Academia para justificar su propio desarrollo intelectual ${ }^{160}$. El nuevo cariz práctico que adopta la idea de una verdad orientada a la operatio será un tema determinante para la reflexión teológica en torno a la salvación humana ${ }^{161}$. Así las cosas, la comprensión novedosa acerca de la verdad, cuya evidencia se acredita en el retorno hacia el Bien trascedente de donde procede el mundo tanto espiritual como material, está no sólo planteada sino incluso sistematizada ya por la filosofía plotiniana $^{162}$.

\footnotetext{
${ }^{156}$ Cf. V 5, 5, 14-27.

157 V 3, 17, 38.

${ }^{158}$ Cf. M. E. Sacchi, «La afirmación de la trascendencia de Dios en la doctrina cristiana y en las conclusiones de la filosofía primera», Sapientia 195-196, Vol. L (1995): 149-183.

${ }^{159}$ Cf. K. Kremer, Die neuplatonische Seinsphilosophie und ihre Wirkung auf Thomas von Aquin (Leiden: Brill, 1966).

${ }^{160}$ Cf. H. Boeder, «¿Por qué motivo ser del ente?», en In officium Sapientiae, trad. y notas por M. Zubiria, Vol. I, 17-45 (Mendoza: UNCuyo-FFyL, 2017), consultada en diciembre 1, 2020, http://bdigital.uncu.edu.ar/objetos_digitales/8889/in-officium-sapientiae-i-corr-.pdf.

${ }^{161}$ Cf. M. Zubiria, Die Teleologie und die Krisis der Principien (Hildesheim-Zürich-New York: Olms, 1995), 52-158.

${ }^{162}$ H. Boeder, «Las concepciones filo-sóficas de la Época Media», In officium Sapientiae, en In officium Sapientiae. Antología de textos filosóficos, trad. y notas por M. Zubiria, Vol. III, 35-58 (Mendoza: UNCuyo-
} 


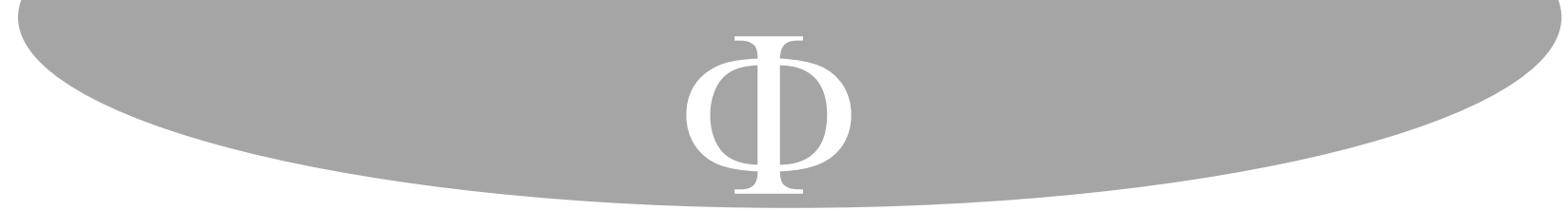

Boulègue, P. Caye, F. Malhomme \& S. Perceau, 273-291. Paris: Classiques Garnier, 2014.

Beierwaltes, W. Denken des Einen. Studien zur neuplatonischen Philosophie und ihrer Wirkungsgeschichte. Frankfurt a. M.: Klostermann, 2016.

Boeder, H. «¿Por qué motivo ser del ente?» y «¿Qué consuma la filosofía primera?». En In officium Sapientiae. Antologia de textos filosóficos. Traducción y notas por M. Zubiria, Vol. I, 17-45, 47-66. Mendoza: UNCuyo-FFyL, 2017. Consultada en diciembre 1, 2020. http://bdigital.uncu.edu.ar/objetos_digitales/8889/in-officium-sapientiae-i-corr.pdf.

Boeder, H. «Las concepciones filo-sóficas de la Época Media». En In officium Sapientiae. Antologia de textos filosóficos. Traducción y notas por M. Zubiria, Vol. III, 35-58. Mendoza: UNCuyo-FFyL, 2017. Consultada en diciembre 1, 2020. http://bdigital.uncu.edu.ar/objetos_digitales/8891/in-officium-sapientiae-iii-corr-.pdf.

Boeder, H. Topología de la Metafísica: la Época Media. Traducción y notas pr M. Zubiria. Pamplona: EUNSA, 2009.

Boissonnault, É. Plotin et ses Contemporains: Union avec l'Un. Québec: Faculté de Philosophie-Université Laval, 2007.

Bord, A. Plotin et Jean de la Croix. Paris: Beauchesne, 1996.

Boyer, Ch. Christianisme et néoplatonisme dans la formation de saint Augustin. Paris: G. Beauchesne, 1920.

Brandâo, B. «Ascensâo e discurso em Plotino». Kriterion Vol. 130 (2014): 515-530.

Bréhier, É. La théorie des incorporels dans l'ancien Stö̈cisme. Paris: Vrin, 1970.

Bréhier, É. La philosophie de Plotin. Paris: Vrin, 1961.

Brisson, L. «Podese Falar de União Mística em Plotino». Kriterion Vol. 116 (2007): 453466.

Burckhardt, J. Del paganismo al cristianismo. La época de Constantino el Grande. Traducido por E. Imaz. México: FCE, 1945.

Burnet, J. Platonis opera Vol. 1-5. Oxford: Clarendon Press, 1900-1907.

Bussanich, J. The One and its relation to Intellect in Plotinus. Leiden-New York: Brill, 1988.

Carabine, D. The Unknown God: negative Theology in the platonic tradition from Plato to Eriugena. Louvain: Peeters Press, 1995.

Charrue, J.-M. Plotin lecteur de Platon. Paris: Les Belles Lettres, 1978.

Ciapalo, R. 'Life' in Plotinus' Explanation of Reality. Chicago: University of ChicagoFaculty of the Graduate School of Loyola, 1987.

Cilento, V. Plotino. Paideia antignostica. Ricostruzione d' un unico scritto da Enneadi III 8, V 8, V 5, II 9. Firenze: Felice le Monnier, 1971.

Coenen, L. et al. Diccionario teológico del Nuevo Testamento. Traducido por M. Sala y A. Herrera, Vol. 1-4. Salamanca: Sígueme, 1990-1994.

Corrigan, K. Reading Plotinus, A Practical Introduction to Neoplatonism. Indiana: Purdue University Press, 2004.

Creuzer, F. \& Moser, G. H. Plotini Enneades cum Marsilii Ficini interpretatione castigata. Paris: Didot, 1896.

Croissant, J. «P. Henry S.J. Le problème de la liberté chez Plotin, dans Revue néoscol. de philos., XXXIII, févr. mai et août 1931, pp. 50-79, 180-215, 318-339». L' Antiquité Classique Vol. 4, 2 (1935): 534-536. 


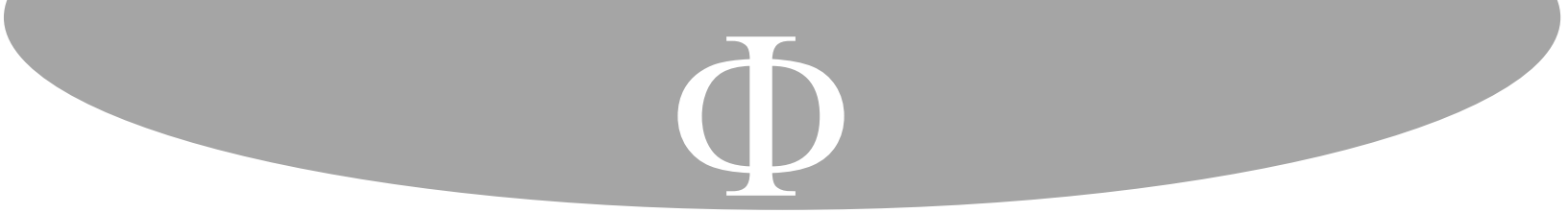

Dillon, J. The Middle Platonists 80 B. C. to A. D. 220. New York: Cornell University Press, 1996.

Dillon, J. «Plotinus at Work on Platonism». Greece \& Rome. Second Series 2, Vol. 39 (1992): 189-204.

Dodds, E. R. Paganos y cristianos en una época de angustia. Madrid: Cristiandad, 1968.

Dodds, E. R. «Tradition and Personal Achievement in the Philosophy of Plotinus». The Journal of Roman Studies, 1-2, Vol. 50 (1960): 1-7.

Dodds, E. R. «The Parmenides of Plato and the Origin of the Neoplatonic One». The Class. Quarterly 3-4, Vol. 22 (1928): 129-142.

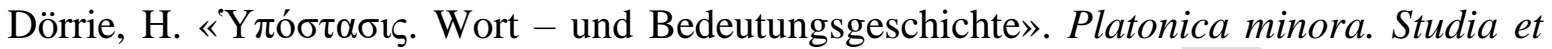
Testimonia Antiqua VIII, 12-69. Munich: W. Fink Verlag, 1976.

Dörrie, H. «Die Frage nach dem Transzendenten im Mittelplatonismus». En Les sources de Plotin. Entretiens sur l'Antiquité Classique V. Editado por E. R. Dodds, 193-241. Genève: Fondation Hardt, 1960.

Festugière, A.-J. La Révélation d'Hermès Trimégiste. IV: Le Dieu Inconnu et la Gnose. Paris: Les Belles Lettres, 1986.

De Gandillac, M. La sagesse de Plotin. Paris: Hachette, 1966.

De Gandillac, M. «Le Plotin de Bergson». Revue de théologie et de philosophie Vol. 23 (1973): 173-183.

García Bazán, F. Oráculos caldeos / Numenio de Apamea. Fragmentos y testimonios. Madrid: Gredos, 2008.

García Bazán, F. «Antecedentes, continuidad y proyecciones del Neoplatonismo». Anuario Filosófico Vol. 33 (2000): 111-149.

García Yebra, V. Ed. Metafísica de Aristóteles. Edición trilingüe. Madrid: Gredos, 1998.

Gatti, M. L. «Plotinus: The Platonic Tradition and the foundation of Neoplatonism». En The Cambridge Companion to Plotinus. Editado por Ll. P. Gerson, 10-37. Cambridge: University Press, 1999.

Gauthier, R.-A. et Jolif, J.-Y. Aristote. L'Éthique à Nicomaque. Louvain-Paris: Publications Universitaires-Béatrice-Nauwelaerts, 1970.

Gerson, Ll. P. Plotinus. London: Routledge, 1994.

Gerson, Ll. P. «Plotinus's Metaphysics: Emanation or Creation?». The Review of Metaphysics 3, Vol. 46 (1993): 559-574.

Guitton, J. Le temps et l'éternité chez Plotin et Saint Augustin. Paris: Boivin et Cie. Éd, 1933. Gutiérrez, R. «El principio de Plotino y el inicio de una época». Arete 1, Vol. II (1990): 4967.

Hadot, P. ¿Qué es la filosofía antigua?. Traducido por I. E. Cazenave Tapie, revisión técnica de M $^{\mathrm{a}}$ I. Santa Cruz. México: FCE, 1998.

Hegel, G. W. F. Fenomenología del espíritu. Edición bilingüe de A. Gómez Ramos. Madrid: Abada, 2010.

Hegel, G. W. F. Lecciones sobre la historia de la filosofía, t. III. Traducido por W. Roces. México-Buenos Aires: FCE, 1955.

Heidegger, M. «¿Qué es esto: la filosofía?» y otros textos breves. Introducción, traducción y notas de M. Zubiria. Mendoza: SS\&CC, 2011.

Henry, P. \& Schwyzer, H.-R. Plotini opera Vol. 1-3. Oxford: University Press, 1964-1982 (ed. minor). 


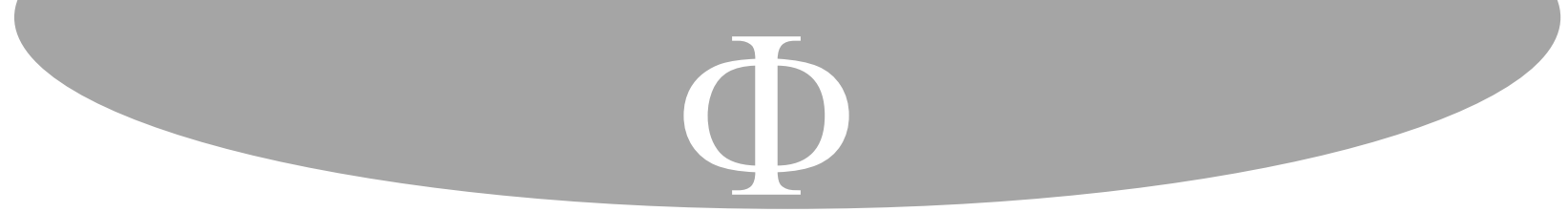

Henry, P. \& Schwyzer, H.-R. Plotini opera Vol. 1-3. Leiden: Brill, 1951-1973 (ed. maior). Ho, P.-Sh. Plotinus' Mystical Teaching of Henosis. An Interpretation in the Light of the Metaphysics of the One. Frankfurt a. M.: Peter Lang, 2015.

Juárez Valero, Nuria. Ontología estética del espacio-luz: arquitecturas noésicas en la filosofía. Tesis doctoral en filosofía. Madrid: UNED, 2018. Consultada en diciembre 1, 2020. http://e-spacio.uned.es/fez/eserv/tesisuned:ED-Pg-FilosofiaNjuarez/JUAREZ_VALERO_NURIA_Tesis.pdf.

Krämer, H.-J. Der Ursprung der Geistmetaphysik. Untersuchungen zur Geschichte des Platonismus zwischen Platon und Plotin. Amsterdam: Schippers, 1964.

Kremer, K. Die neuplatonische Seinsphilosophie und ihre Wirkung auf Thomas von Aquin. Leiden: Brill, 1966.

Lafont, G. Histoire théologique de l'Église catholique. Itinéraire et formes de la théologie. Paris: Cerf, 1994.

López Eire, A. «Plotino frente a sus fuentes». Boletín del Instituto de Estudios Helenísticos 1, Vol. 7 (1973): 65-77.

Martino, G. «Filosofía y exégesis en las Enéadas. Las alas del alma plotiniana en la lectura del Fedro platónico». Areté. Revista de Filosofía 1, Vol. XXVI (2014): 77-108.

Martino, G. «Mística y exégesis en la filosofía de Plotino». NOVA TELLVS 2, Vol. 30 (2012): 73-98.

Martino, G. «La mística plotiniana: experiencia, doctrina e interpretación». Revista Archai Vol. 5 (2010): 67-76.

Merlan, Ph. From Platonism to Neoplatonism. The Hague: Martinus Nijhoff, 1968.

Minio-Paluello, L. Aristotelis Categoriae et Liber de Interpretatione. Oxford: Clarendon Press, 1949.

Moreau, J. Plotin ou la gloire de la philosophie antique. Paris: Vrin, 1970.

Moreschini, C. y Norelli, E. Patrología. Manual de literatura cristiana antigua griega y latina. Salamanca: Sígueme, 2009.

Numénius. Fragments, Éd. trad. française et comm. par É. des Places. Paris: Les Belles Lettres, 1973.

Olivieira, L. «Notas sobre Lógica e Dialética na Enéada de Plotino». Trans/Form/Acao 2, Vol. 30 (2007): 167-178.

O’ Brien, D. «Le volontaire et la nécessité: réflexions sur la descente de l'âme dans la philosophie de Plotin». Revue Philosophique de la France et de l'Étranger 4, Vol. 167 (1977): 401-422.

Pigler, A. Plotin une Métaphysique de l'amour. L'amour comme structure du monde intelligible. Vrin: Paris, 2002.

Pigler, A. «Plotin exégète de Platon ? La question du temps». Revue Philosophique de la France et de l'Étranger 1, Vol. 186 (1996): 107-117.

Plotin. Ennéades. Editado y traducipor por É. Bréhier, Vol. 1-6. Paris: Les Belles Lettres, 1924-1938.

Plotino. Enneadi. Traducción y comentario crítico por V. Cilento, Vol. 1-3. Bari: Gius. Laterza \& Figli, 1947-1949.

Plotinus. The Six Enneads. Traducido por S. MacKenna \& B. S. Page. Chicago: University of Chicago, Encyclopaedia Britannica, 1952. 


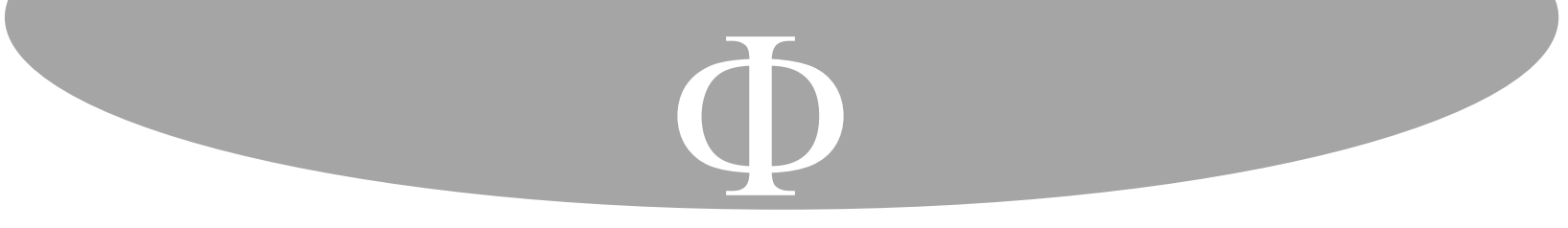

Porfirio y Plotino. Vida de Plotino y Enéadas I-II, III-IV, V-VI. Introducción, traducción y notas por J. Igal Alfaro. Madrid: Gredos, 1982-1998.

Porphyry and Plotinus. On the life of Plotinus and Enneads in Seven Volumes. Traducido por A. H. Armstrong. Cambridge-Massachusetts: Harvard University Press, 1966-1989.

Porro, P. «Heidegger, la filosofia medievale, la medievistica contemporanea». Quaestio Vol. 1 (2001): 431-462.

Praechter, K. «Die griechischen Aristoteleskommentare». ByZ (1909): 516-538.

Puech, H.-Ch. «Plotin et les Gnostiques». En Les sources de Plotin. Entretiens sur l'Antiquité Classique V. Editado por E. R. Dodds, 159-174. Genève: Fondation Hardt, 1960.

Quispel, G. Gnosis als Weltreligion. Zürich: Origo Verlag, 1951.

Radice, R. Lexicon II Plotinus. Milano: Università Vita-Salute S. Raffaele, 2004.

Reale, G. «Fundamentos, estructura dinámico-relacional y caracteres esenciales de la metafísica de Plotino». Anuario Filosófico Vol. 33 (2000): 163-191.

Rist, J. M., Plotinus: The Road to Reality. Cambridge: University Press, 1967.

Rist, J. M. «Plotinus and Christian philosophy». En The Cambridge Companion to Plotinus. Editado por Ll. P. Gerson, 386-414. Cambridge: University Press, 1999.

Ritter, A. R. «Platonismus und Christentum in der Spätantike». Theologische Rundschau Vol. 49 (1984): 31-56.

Romano, F. Il neoplatonismo. Roma: Carocci, 1998.

Saffrey, H.-D. Recherches sur le Néoplatonisme après Plotin. Paris: Vrin, 1990.

Sacchi, M. E. «La afirmación de la trascendencia de Dios en la doctrina cristiana y en las conclusiones de la filosofía primera». Sapientia, 195-196, Vol. L (1995): 149-183.

Santa Cruz, Ma. I. «Modos de conocimiento en Plotino». Estudios de Filosofía Vol. 34 (2006): 201-216.

Santa Cruz, Ma. I. «Plotino y el neoplatonismo». En Historia de la Filosofía Antigua. Editado por C. García Gual, 339-361. Madrid: Enciclopedia Iberoamericana, 1997.

Schiller, J. P. «Plotinus and Greek Rationalism». Apeiron: A Journal for Ancient Philosophy and Science 1, Vol. 12 (1978): 37-50.

Sleeman, J. H. \& Pollet, G. Lexicon Plotinianum. Leiden-Leuven: Brill, 1980.

Sorabji, R. Self, Ancient and Modern Insights about Individuality, Life and Death. Chicago: The University of Chicago Press, 2006.

Soto-Bruna, Ma. J. «El neoplatonismo». En Historia universal del pensamiento filosófico. Editado por A. S. Naya, Vol. I, 587-598. Vizcaya: Ortuella, 2007.

Soto-Bruna, Ma. J. «Causalidad, expresión y alteridad. Neoplatonismo y modernidad». Anuario Filosófico Vol. 33 (2000): 533-554.

von Stein, H. Sieben Bücher zur Geschichte des Platonismus. Göttingen: Vandenhoeck \& Ruprecht, 1864.

Szlezák, Th. A. Platon und Aristoteles in der Nuslehre Plotins. Basel-Stuttgart: Schwabe \& Co, 1979.

Ullmann, R. A. Plotino, Um estudo das Enéadas. Porto Alegre: edipucrs, 2002.

Whittaker, J. «EПEKEINA NOY KAI OY $I A S »$. Vigilia Christianae 2, Vol. 23 (1969): 91104.

Zamora Calvo, J. Ma. «¿Cómo elevarse a lo divino? Sobre las últimas palabras de Plotino». Synthesis 1, Vol. 25 (2018). Consultada en diciembre 1, 2020. https://doi.org/10.24215/1851779Xe032. 


\section{$\Phi$}

Zamora Calvo, José Ma. «La noción de procesión en Plotino». Areté. Revista de Filosofía 1, Vol. IX (1997): 85-105.

Zubiria, M. El amor sapientiae ante la diferencia absoluta (Plotino, VI 9). Mendoza: SS\&CC, 2012.

Zubiria, M. Die Teleologie und die Krisis der Principien. Hildesheim-Zürich-New York: Olms, 1995.

Zubiria, M. «Dificultades en la concepción hegeliana de la historia de la filosofía». Diálogos Vol. 62 (1993): 73-86.

Enviado: 13 de diciembre de 2020 Aceptado: 7 de febrero de 2021 